\title{
Captured on (bio)film
}

Transmission of human T lymphotrophic virus 1 ( $\mathrm{HTLV}-1)$ occurs at transient points of cell-to-cell contact between T cells. At these points, known as virological synapses, viral particles are thought to bud from the infected cell and fuse with the membrane of the uninfected cell; however, a detailed understanding of the localization of viral components and the molecular mechanisms underlying this form of transmission have been lacking. In an article published in Nature Medicine, Thoulouze

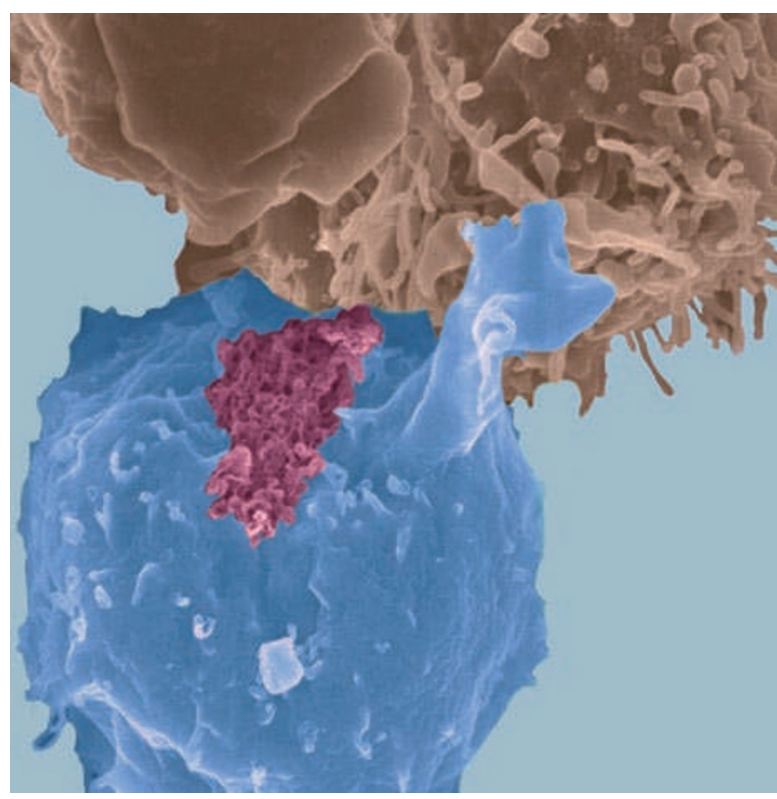

Scanning electron micrograph showing the biofilm-like structures (magenta) that are formed by human T lymphotrophic virus 1 particles, adhere to the surface of infected T cells (brown) and are transferred to target cells (blue) on cell-to-cell contact. Image courtesy of M. I. Thoulouze, the Pasteur Institute, Paris, France. and colleagues now show that HTLV-1 particles form biofilm-like structures that adhere to the surface of infected T cells and mediate rapid transfer of virus to the surface of uninfected cells.

Using confocal and electron microscopy of primary $\mathrm{CD} 4^{+} \mathrm{T}$ cells isolated from HTLV-1-infected individuals, the authors observed that HTLV-1 particles form discrete clusters at the cell surface. These clusters, which may be localized at the edge of membrane extensions, contained virus particles surrounded by a smooth, electron-dense material. Extracellular matrix (ECM) components are known to be important for HTLV-1 attachment to the cell surface during infection, leading the authors to investigate whether the ECM may also have a role in anchoring and concentrating virus particles into extracellular assemblies following viral budding. They found that the HTLV-1 clusters could be stained with a range of lectins, indicating the presence of carbohydrate components. Furthermore, the clusters were also found to be enriched for several ECM proteins, many of which are overexpressed or relocalized in HTLV-1-infected cells. Proteins that were enriched in these biofilm structures include collagen, agrin, galectin 3 and tetherin, whereas other ECM proteins, such as fibronectin and galectin 1, were not enriched, indicating that the composition of proteoglycans in the biofilms is specific.
Thoulouze and colleagues observed that infected $\mathrm{T}$ cells formed tight virological synapses with uninfected cells and that transfer of HTLV-1 clusters to uninfected cells was rapid and was accompanied by transfer of ECM components. Three-dimensional imaging of the synapses revealed that the clusters bridged the gap between infected and uninfected cells at the margins of the virological synapse. Finally, the authors used mechanical or heparin washes to disrupt interactions in the ECM and remove the HTLV-1 clusters from the $\mathrm{T}$ cell surface, leading to an $80 \%$ decrease in the capacity of the washed cells to infect other T cells.

Such structures may enable HTLV-1 to avoid immune recognition, to maintain infectious titres by concentrating viral particles at the transmission site and to resist disruption by shear forces in a physiological setting. It will be interesting to see whether other viruses form similar extracellular clusters with biofilm-like properties.

Andrew Jermy

ORIGINAL RESEARCH PAPER Pais-Correia,

A. M. et al. Biofilm-like extracellular viral assemblies mediate HTLV-1 cell-to-cell transmission at virological synapses. Nature Med. 20 Dec 2009 (doi:10.1038/nm.2065)

FURTHER READING Sattentau, Q. Avoiding the void: cell-to-cell spread of human viruses. Nature Rev. Microbiol. 6, 815-826 (2008) 